\section{Efetividade do aconselhamento nutricional da Pastoral da Criança sobre a variação de hemoglobina entre menores de seis anos de idade}

\author{
Effectiveness of nutritional counseling \\ provided by the Children's Mission on \\ hemoglobin variation in under-six children
}

Iná dos Santos 1

Juraci de Almeida Cesar 2

Gicele Minten 1

Paula Lobo Marco 1

Neiva Valle 1

\footnotetext{
1 Centro de Pesquisas

Epidemiológicas,

Universidade Federal

de Pelotas, Pelotas, Brasil.

2 Faculdade de Medicina

Fundação Universidade

de Rio Grande,

Rio Grande, Brasil.

Correspondência

I. Santos

Programa de Pós-graduação em Epidemiologia, Centro de Pesquisas Epidemiológicas,

Universidade Federal de

Pelotas. C. P. 464, Pelotas, RS

96001-970, Brasil.

inasantos@uol.com.br
}

\begin{abstract}
A community intervention was conducted to evaluate the effectiveness of leadership training in the Children's Mission (of the Brazilian Catholic Church) for providing nutritional counseling on hemoglobin variation in children less than six years of age. Two areas of activity by the Children's Mission in Pelotas, Rio Grande do Sul State, Brazil, were randomly selected and the leaders in one were trained (intervention group). The other area constituted the control group. After providing consent, mothers were interviewed concerning the family and the child's health and eating. Hemoglobin was measured with a portable photometer upon entry into the study and at six months. The outcome variable was hemoglobin variation between the first and second measurements. The study included 183 intervention children and 179 controls, comparable as to sex, age, characteristics at birth, iron supply, and mean hemoglobin. In the adjusted analysis, variation in the intervention group was $0.18 \pm 0.27 \mathrm{~g} /$ dl greater than in the control group. Although the difference between the two groups was not statistically significant, the variation was positive in the intervention group and negative in the control group, suggesting a beneficial effect from the training.
\end{abstract}

Iron Deficiency; Iron-Deficiency Anaemia; Child Nutrition

\section{Introdução}

A deficiência de ferro é, isoladamente, a mais comum das deficiências nutricionais encontradas no mundo. A anemia constitui sua forma de apresentação mais grave, estando associada com risco aumentado de morrer 1, maior susceptibilidade a infecções 2 , déficit do desenvolvimento cognitivo e psicomotor 3 e problemas de comportamento 4 .

Três fatores contribuem para a alta prevalência de anemia na infância: as reservas de ferro ao nascer, a velocidade de crescimento e o balanço entre a ingestão e as perdas do mineral. As principais causas de anemia são o baixo consumo de ferro e sua baixa biodisponibilidade nos alimentos que compõem a dieta na infância. A amamentação exclusiva até os seis meses de idade, a fortificação da farinha de trigo ou do leite, a suplementação com sais de ferro e a educação nutricional são as estratégias geralmente utilizadas na prevenção da anemia na infância 5 .

$\mathrm{O}$ atual estudo teve como objetivo avaliar a efetividade do treinamento das líderes da Pastoral da Criança em aconselhamento nutricional, a ser dirigido às mães, sobre a variação no nível de hemoglobina entre menores de seis anos de idade. Em saúde pública, estudos de efetividade, em que nenhuma medida subseqüente é tomada para garantir a adesão, tanto por parte dos que recebem quanto dos profis- 
sionais que executam a intervenção, são importantes para avaliar o impacto de intervenções que se mostraram eficazes quando testadas nas condições ideais dos ensaios randomizados 6 .

\section{Material e métodos}

Este foi um estudo experimental, do tipo intervenção comunitária, controlada e unicega. Inicialmente foram localizadas todas as comunidades de Pelotas, Rio Grande do Sul, Brasil, atendidas pela Pastoral da Criança. Com base nesse mapeamento, foram definidas duas áreas geográficas não contíguas, cada uma delas compreendendo três comunidades, com um número aproximadamente igual de crianças cobertas pela Pastoral da Criança. Por sorteio (lançamento de uma moeda), uma das áreas foi selecionada para ser o Grupo Intervenção. A outra área passou a constituir o Grupo Controle. As líderes que trabalhavam na área de intervenção $(n=10)$ foram treinadas em aconselhamento nutricional e as do grupo controle $(n=13)$ continuaram seu trabalho de rotina.

As líderes do grupo intervenção foram treinadas para avaliar a alimentação das crianças e a aconselhar as mães no uso de alimentos locais, de preço acessível para populações de baixa renda, ricos em macro e micronutrientes, cuja aceitabilidade e eficácia no crescimento infantil já haviam sido testadas em estudos anteriores 7,8 . O conteúdo do treinamento foi produzido localmente por meio da tradução e adaptação das normas da Organização Mundial da Saúde (OMS) e Fundo das Nações Unidas para a Infância (UNICEF) 9,10, dentro da estratégia de Atenção Integrada às Doenças Prevalentes na Infância. Todo o material escrito, originalmente adaptado para o treinamento de médicos em atenção primária à saúde, foi readaptado para adequar-se ao perfil educacional das líderes. Um vídeo sobre amamentação produzido pela OMS/UNICEF e o Cartão da Mãe, resumindo todas as recomendações e organizado de acordo com a idade da criança 11 , foram utilizados no treinamento das líderes. Resumidamente, a intervenção consistiu no incentivo à amamentação para crianças de até seis meses de idade e no aconselhamento quanto ao tipo e freqüência de consumo de alimentos ricos em macro e micronutrientes (papa grossa, ao invés de sopa rala; carne de galinha desfiada ou de rês moída; fígado de galinha; grão de feijão, ao invés de apenas o caldo; gema de ovo; e vegetais de folhas verdes). $\mathrm{O}$ treinamento consistiu em leitura do material, discussão em grupo das recomendações nutricionais, exercícios programados e dramatizações. Inadvertidamente, duas líderes de uma das comunidades controle receberam o treinamento, sendo as crianças por elas acompanhadas excluídas do estudo.

Considerando de 1,6g/dl o desvio padrão da média do nível de hemoglobina entre crianças de 6 a 59 meses de idade 12, o estudo foi planejado para detectar, com um poder de $80,0 \%$, uma diferença de $0,5 \mathrm{~g} / \mathrm{dl}$ no nível de hemoglobina entre os dois grupos, significativa ao nível de $95 \%$. Acrescentando-se $10,0 \%$ para perdas, recusas e controle de fatores de confusão, seriam necessárias 177 crianças em cada grupo.

O trabalho de campo foi realizado por quatro entrevistadoras previamente treinadas que visitavam crianças nos domicílios. Após consentimento informado, entrevistavam a mãe e examinavam a criança. Em cada residência eram incluídas todas as crianças da faixa etária do estudo, desde que atendidas pela Pastoral da Criança. As crianças foram visitadas duas vezes (ao ingressar no estudo e seis meses mais tarde). A Figura 1 apresenta o fluxograma do estudo.

Um questionário estruturado, pré-codificado, foi utilizado para a entrevista com a mãe ou, na ausência desta, com o responsável pela criança. Na primeira visita, as mães foram inquiridas quanto a características demográficas e sócio-econômicas da família: idade; escolaridade e trabalho atual da mãe; pai ou padrasto vivendo no mesmo domicílio; escolaridade e trabalho do pai ou padrasto; moradores do domicílio; renda familiar mensal; classe social obtida pelo escore da Associação Nacional de Empresas de Pesquisa (Critério de classificação econômica Brasil. http://www.anep.org.br, acessado em 12/Fev/2003), compreendendo a escolaridade da pessoa com maior renda do domicílio, número de rádios na residência, posse de geladeira, máquina de lavar roupas e televisão em cores; e, disponibilidade, dentro de casa, de água encanada e de sanitário com descarga).

Sobre a criança investigaram-se as características demográficas (sexo, cor da pele e idade em meses), morbidades (diarréia no dia da entrevista e nos últimos 15 dias; tosse com ou sem febre; dispnéia ou congestão nasal nos últimos sete dias; número de hospitalizações; diagnóstico atual ou passado de anemia feito por médico; uso atual de medicamentos; verificação da embalagem dos medicamentos em uso para 


\section{Figura 1}

Fluxograma do estudo. Pastoral da Criança, Pelotas, Rio Grande do Sul, Brasil, 2003
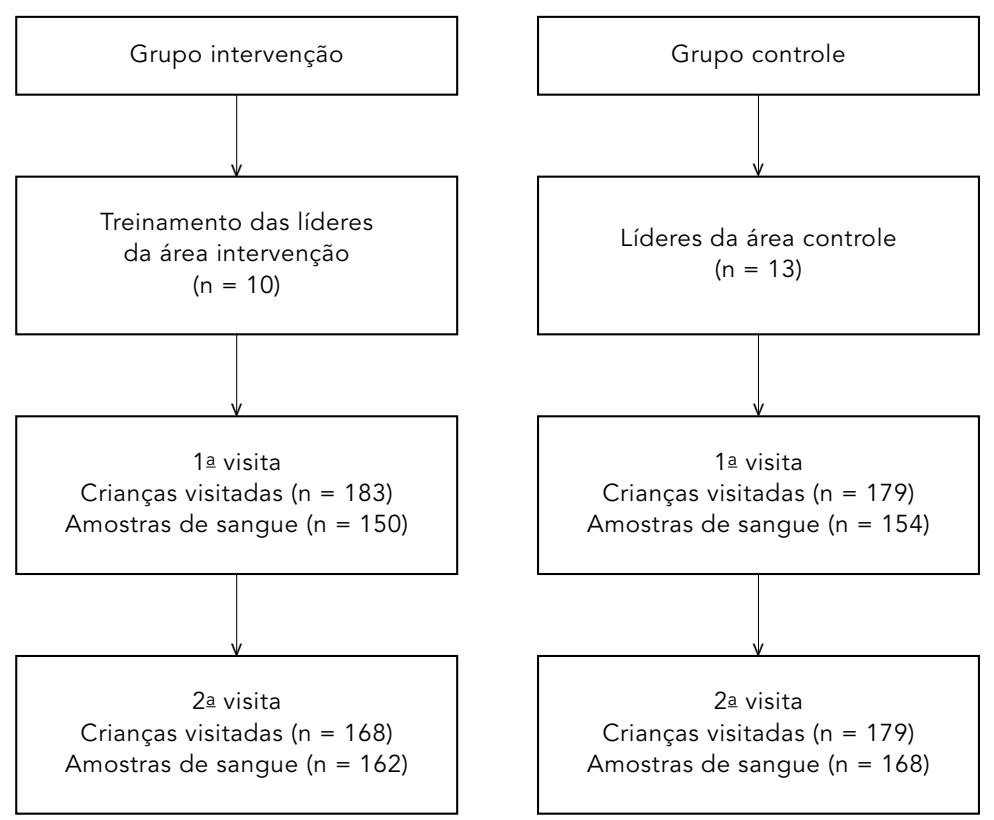

checar consumo atual de sais de ferro; e estado vacinal de acordo com a idade), hábitos alimentares (amamentação atual entre os menores de três anos de idade; amamentação em algum momento após o nascimento; consumo semanal de carne, fígado de ave, ovo, multimistura, adição de uma colher das de chá de óleo ao prato da criança e, para os menores de oito meses de idade, consumo semanal de papa grossa), características de nascimento (prematuridade, peso e comprimento ao nascer) e estado nutricional atual (peso e altura ou comprimento, a partir dos quais calcularam-se os escores $\mathrm{Z}$ de peso para idade, comprimento/altura para idade e peso para altura, tendo como referência a população do National Center of Health Statistics).

Um recordatório alimentar das 24 horas anteriores à entrevista foi obtido nas duas visitas. Com o recordatório, coletou-se informação detalhada sobre o modo de preparo dos alimentos, a quantidade consumida pela criança em medidas caseiras e, posteriormente, traduziuse esse consumo em unidades de macronutrientes e de ferro.

Sobre a líder da Pastoral da Criança, investigou-se se havia visitado a criança no último mês, se na última visita entrou na casa, viu a criança, aconselhou a mãe sobre alimentação e convidou para o Dia da Celebração da Vida, e havendo a mãe comparecido às atividades do Dia da Celebração da Vida, se a criança foi pesada e recebeu lanche, se a líder falou sobre saúde e se a mãe ficou até o final da pesagem de todas as crianças.

O nível de hemoglobina em gramas por decilitro ( $\mathrm{g} / \mathrm{dl}$ ) foi obtida pela leitura direta em fotômetro portátil (hemoglobinômetro HemoCue) de amostra de sangue capilar da polpa digital colhido em microcuvetas. Considerouse anemia o nível de hemoglobina inferior a $11,0 \mathrm{~g} / \mathrm{dl}$, conforme recomendação da OMS 13 . O desfecho investigado foi a diferença entre os dois grupos (intervenção e controle) quanto à variação média do nível de hemoglobina da primeira para a segunda medida.

$\mathrm{Na}$ análise, os grupos foram, inicialmente, comparados quanto a distribuição das potenciais variáveis de confusão. Conforme o caso, o teste qui-quadrado $\left(\chi^{2}\right)$ ou o teste $\mathrm{t}$ foram usados nessas comparações. O efeito da intervenção sobre a variação dos níveis de hemoglobina da primeira para a segunda visita foi analisada por regressão linear por meio de modelagem de multiníveis. Quatro níveis foram considerados para essa análise. $O$ primeiro, cranealmente, representado pelas comunidades de residência das crianças; o segundo, pelas líderes; o terceiro, pelas mães; e por último, as crianças. O pacote estatístico MLn foi utilizado nessa análise. Foram levadas para a análise multivariada as variáveis que se associaram com ambos, o status de intervenção ou controle e com o desfecho, em um nível de significância de $\mathrm{p}<0,20$. Todas as análises foram conduzidas por intenção de tratar.

O protocolo do estudo foi submetido ao Comitê de Ética da Faculdade de Medicina da Universidade Federal de Pelotas. Cada criança somente foi incluída no estudo após a obtenção do consentimento informado e por escrito da mãe ou responsável. Na segunda visita, as mães receberam o resultado dos dois exames por escrito, sendo orientadas a apresentá-los no Posto de Saúde mais próximo de sua residência.

\section{Resultados}

Foram incluídas no estudo 362 crianças, 183 no grupo intervenção e 179 no controle. Na Tabela 1, a proporção de crianças de cor branca foi significativamente maior no grupo intervenção do que no controle (respectivamente, $83,5 \% \mathrm{e}$ $69,8 \% ; \mathrm{p}=0,01$ ). A média de idade e a distribui- 
Características demográficas das crianças e características demográficas e sócio-econômicas das famílias estudadas. Pastoral da Criança, Pelotas, Rio Grande do Sul, Brasil, 2003.

\begin{tabular}{|c|c|c|c|c|c|}
\hline \multirow[t]{2}{*}{ Características } & \multicolumn{2}{|c|}{ Grupo intervenção } & \multicolumn{2}{|c|}{ Grupo controle } & \multirow[t]{2}{*}{$\mathbf{p}$} \\
\hline & $n=18$ & $\%$ & $\mathrm{n}=17$ & $\%$ & \\
\hline Sexo & & & & & 0,50 \\
\hline Masculino & 88 & 48,1 & 93 & 52,0 & \\
\hline Feminino & 95 & 51,9 & 86 & 48,0 & \\
\hline Cor & & & & & 0,01 \\
\hline Branca & 152 & 83,5 & 125 & 69,8 & \\
\hline Preta & 18 & 9,9 & 29 & 16,2 & \\
\hline Parda & 9 & 4,9 & 15 & 8,4 & \\
\hline Outra & 3 & 1,6 & 10 & 5,6 & \\
\hline Idade (em meses)* & \multicolumn{2}{|c|}{$34,9 \pm 19,4$} & \multicolumn{2}{|c|}{$37,6 \pm 18,2$} & 0,10 \\
\hline$<12$ & 28 & 15,3 & 16 & 8,9 & 0,50 \\
\hline $12-23,9$ & 30 & 16,4 & 27 & 15,1 & \\
\hline $24-35,9$ & 36 & 19,7 & 38 & 21,2 & \\
\hline $36-47,9$ & 35 & 19,1 & 42 & 23,5 & \\
\hline $48-59,9$ & 30 & 16,4 & 30 & 16,8 & \\
\hline $60-71,9$ & 24 & 13,1 & 26 & 14,5 & \\
\hline Idade materna (em anos)* & \multicolumn{2}{|c|}{$29,8 \pm 8,5$} & \multicolumn{2}{|c|}{$29,2 \pm 7,5$} & 0,40 \\
\hline$<20$ & 10 & 5,5 & 15 & 8,4 & 0,70 \\
\hline $20-29$ & 89 & 48,6 & 81 & 45,3 & \\
\hline $30-39$ & 66 & 36,1 & 65 & 36,3 & \\
\hline$\geq 40$ & 18 & 9,8 & 18 & 10,1 & \\
\hline Escolaridade mãe (em anos)* & \multicolumn{2}{|c|}{$4,8 \pm 2,6$} & \multicolumn{2}{|c|}{$4,4 \pm 2,6$} & 0,09 \\
\hline 0 & 8 & 4,4 & 12 & 6,7 & $0,03 * \star \star$ \\
\hline $1-4$ & 79 & 43,2 & 93 & 52,0 & \\
\hline $5-8$ & 82 & 44,8 & 64 & 35,8 & \\
\hline $9-11$ & 14 & 7,7 & 10 & 5,6 & \\
\hline Escolaridade pai/padrasto (em anos) ${ }^{\star \star}$ & \multicolumn{2}{|c|}{$4,4 \pm 2,7$} & \multicolumn{2}{|c|}{$3,8 \pm 2,3$} & 0,03 \\
\hline 0 & 16 & 11,2 & 12 & 8,3 & 0,02 \\
\hline $1-4$ & 54 & 37,8 & 78 & 53,8 & \\
\hline $5-13$ & 73 & 51,0 & 55 & 37,9 & \\
\hline Menores de seis anos & \multicolumn{2}{|c|}{$1,7 \pm 0,8$} & \multicolumn{2}{|c|}{$1,9 \pm 0,9$} & 0,04 \\
\hline \multicolumn{6}{|l|}{ Classe social ${ }^{\star \star \star \star}$} \\
\hline $\mathrm{D}$ & 77 & 53,8 & 51 & 35,2 & 0,002 \\
\hline $\mathrm{E}$ & 66 & 46,2 & 94 & 64,8 & \\
\hline Renda familiar mensal (em reais)* & \multicolumn{2}{|c|}{$373,96 \pm 304,99$} & \multicolumn{2}{|c|}{$221,58 \pm 147,78$} & $<0,001$ \\
\hline $0-150$ & 28 & 17,3 & 52 & 33,8 & $<0,001$ \\
\hline $151-240$ & 36 & 22,2 & 43 & 27,9 & \\
\hline $241-375$ & 42 & 25,9 & 38 & 24,7 & \\
\hline $376-1.750$ & 56 & 34,6 & 21 & 13,6 & \\
\hline Água encanada dentro de casa & & & & & 0,05 \\
\hline $\operatorname{Sim}$ & 167 & 93,3 & 151 & 86,8 & \\
\hline Não & 12 & 6,7 & 23 & 13,2 & \\
\hline Tipo de privada & & & & & 0,008 \\
\hline Com descarga & 148 & 88,6 & 123 & 77,4 & \\
\hline Sem descarga & 19 & 11,4 & 36 & 22,6 & \\
\hline
\end{tabular}

* média \pm desvio padrão; ${ }^{*}$ somente para pais/padrastos que moram no domicílio da criança;

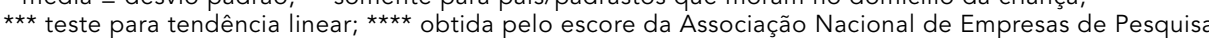

(Critério de classificação econômica Brasil. http://www.anep.org.br, acessado em 12/Fev/2003). 
ção das crianças por faixa etária e por sexo foram semelhantes entre os grupos. No grupo intervenção, o número de pais com maior escolaridade foi mais elevado do que no controle. Embora o número médio de moradores por domicílio e a proporção de famílias extensivas fossem semelhantes, o número médio de crianças menores de seis anos foi maior no grupo controle $(\mathrm{p}=0,04)$. Quanto à classe social, todas as crianças pertenciam a famílias dos dois extratos sociais mais pobres, sendo que $53,8 \%$ das do grupo intervenção, contra $35,2 \%$ das do controle, estavam na classe D $(p=0,002)$. Consistente com esse achado, a renda familiar mensal no grupo intervenção foi, em média, $\mathrm{R} \$ 152,38$ maior do que no controle ( $\mathrm{p}<0,001)$. A diferença quanto ao destino dos dejetos foi estatisticamente significativa ( $p=0,008)$, sendo que sanitário com descarga foi referido por $88,6 \%$ das famílias do grupo intervenção, contra $77,4 \%$ das do grupo controle.

O número de crianças apresentando diarréia no dia da entrevista ou no período de 15 dias anteriores não diferiu entre os grupos (Tabela 2). A presença do sintoma "tosse" sem outros sintomas foi menos freqüente no grupo in- tervenção do que no controle $(\mathrm{p}=0,02)$. A proporção de crianças com história de duas ou mais hospitalizações foi duas vezes menor no grupo intervenção $(p=0,005)$. Foi freqüente nos dois grupos o relato de diagnóstico médico de anemia atual ou no passado. Mais de $1 / 5$ das crianças do grupo intervenção $(n=42)$ e $15,6 \%(n=28)$ das do grupo controle estavam em uso de algum medicamento no momento da entrevista $(p=0,08)$. No grupo intervenção, $58,5 \%(n=24)$ dessas recebiam sulfato ferroso, contra $41,4 \%(n=12)$ no controle $(p=0,20)$. A prevalência de prematuridade e baixo peso ao nascer, bem como o peso médio ao nascer foram semelhantes entre os grupos intervenção e controle (respectivamente, $10,4 \%$ versus $14,5 \%$, $\mathrm{p}=0,20 ; 12,0 \%$ versus $11,7 \%, \mathrm{p}=1,00 ;$ e $3.187,13 \pm$ $619,92 \mathrm{~g}$ versus $3.103,85 \pm 532,21 \mathrm{~g}, \mathrm{p}=0,10$ ).

O consumo de calorias, proteínas, lipídeos, hidratos de carbono e ferro, nas 24 horas que precederam a entrevista, foi semelhante entre os grupos (Tabela 3). Foi alto o relato de consumo semanal de carne em ambos os grupos ( $\mathrm{p}=$ 0,40). Menos de 1/3 das crianças, nos dois grupos, consumia fígado de ave pelo menos uma vez por semana. Entre as consumidoras, não

Tabela 2

Prevalência ponto e prevalência no período de morbidades comuns na infância e estado vacinal das crianças Pastoral da Criança, Pelotas, Rio Grande do Sul, Brasil, 2003.

\begin{tabular}{|c|c|c|c|c|c|}
\hline \multirow[t]{2}{*}{ Variável } & \multicolumn{2}{|c|}{ Grupo intervenção } & \multicolumn{2}{|c|}{ Grupo controle } & \multirow[t]{2}{*}{$\mathrm{p}$} \\
\hline & $n=183$ & $\%$ & $n=179$ & $\%$ & \\
\hline Diarréia no dia da entrevista & 5 & 2,7 & 8 & 4,5 & 0,40 \\
\hline Diarréia nos últimos 15 dias & 14 & 7,7 & 19 & 10,6 & 0,30 \\
\hline Tosse nos últimos 7 dias & 73 & 39,9 & 93 & 52,0 & 0,02 \\
\hline Tosse + febre* & 21 & 28,8 & 19 & 20,4 & 0,20 \\
\hline Tosse + dispnéia* & 36 & 49,3 & 38 & 40,9 & 0,30 \\
\hline Tosse + congestão nasal* & 41 & 56,2 & 54 & 58,1 & 0,80 \\
\hline História de hospitalizações ${ }^{\star \star}$ & \multicolumn{2}{|c|}{$0,60 \pm 1,10$} & \multicolumn{2}{|c|}{$1,10 \pm 1,80$} & 0,002 \\
\hline 0 & 108 & 59,0 & 89 & 49,7 & 0,005 \\
\hline 1 & 54 & 29,5 & 46 & 25,7 & \\
\hline$\geq 2$ & 21 & 11,5 & 44 & 24,6 & \\
\hline $\begin{array}{l}\text { Diagnóstico médico de anemia atual } \\
\text { ou no passado }\end{array}$ & 88 & 49,4 & 101 & 57,4 & 0,10 \\
\hline Relato de anemia atual & 13 & 7,6 & 14 & 8,4 & 0,80 \\
\hline Tomando algum medicamento & 42 & 23,0 & 28 & 15,6 & 0,08 \\
\hline 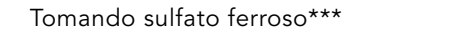 & 24 & 58,5 & 12 & 41,4 & 0,20 \\
\hline Estado vacinal completo para a idade & 146 & 89,0 & 148 & 86,5 & 0,50 \\
\hline
\end{tabular}

* somente entre os que referiram tosse nos últimos sete dias; ** média \pm desvio padrão;

$\star \star \star$ somente entre os que estavam em uso de algum medicamento. 
Alimentação conforme recordatório alimentar de 24 horas e freqüência semanal de consumo de alguns alimentos e estado nutricional das crianças. Pastoral da Criança, Pelotas, Rio Grande do Sul, Brasil, 2003.

\begin{tabular}{|c|c|c|c|c|c|}
\hline \multirow[t]{2}{*}{ Variável } & \multicolumn{2}{|c|}{ Grupo intervenção } & \multicolumn{2}{|c|}{ Grupo controle } & \multirow[t]{2}{*}{$\mathbf{p}$} \\
\hline & $\mathrm{n}=18$ & $\%$ & $\mathrm{n}=17$ & $\%$ & \\
\hline \multicolumn{6}{|l|}{ Recordatório alimentar 24 horas* } \\
\hline Kcal & \multicolumn{2}{|c|}{$1.284,78 \pm 582,61$} & \multicolumn{2}{|c|}{$1.351,51 \pm 715,07$} & 0,30 \\
\hline Proteínas (mg) & \multicolumn{2}{|c|}{$50,52 \pm 27,08$} & \multicolumn{2}{|c|}{$51,27 \pm 26,28$} & 0,80 \\
\hline Lipídeos & \multicolumn{2}{|c|}{$42,16 \pm 24,04$} & \multicolumn{2}{|c|}{$45,35 \pm 36,67$} & 0,30 \\
\hline Hidratos de carbono & \multicolumn{2}{|c|}{$172,05 \pm 79,28$} & \multicolumn{2}{|c|}{$182,58 \pm 90,38$} & 0,20 \\
\hline Ferro & \multicolumn{2}{|c|}{$8,45 \pm 6,07$} & \multicolumn{2}{|c|}{$8,42 \pm 5,22$} & 0,90 \\
\hline \multicolumn{6}{|l|}{ Consumo semanal } \\
\hline Carne & 143 & 79,4 & 147 & 83,1 & 0,40 \\
\hline Fígado & 40 & 23,0 & 48 & 28,2 & 0,20 \\
\hline Gema & 96 & 55,2 & 84 & 48,3 & 0,20 \\
\hline Multimistura & 23 & 12,6 & 4 & 2,2 & 0,00 \\
\hline Uma colher chá óleo no prato & 1 & 0,5 & 7 & 3,9 & 0,03 \\
\hline Papa grossa** & 3 & 25,0 & 3 & 37,5 & 0,60 \\
\hline \multicolumn{6}{|l|}{ Freqüência semanal (em dias) ${ }^{\star \star \star}$} \\
\hline Carne & \multicolumn{2}{|c|}{$4,70 \pm 2,40$} & \multicolumn{2}{|c|}{$4,30 \pm 2,50$} & 0,10 \\
\hline Fígado & \multicolumn{2}{|c|}{$1,70 \pm 0,80$} & \multicolumn{2}{|c|}{$1,90 \pm 1,40$} & 0,30 \\
\hline Gema & \multicolumn{2}{|c|}{$2,30 \pm 1,50$} & \multicolumn{2}{|c|}{$2,10 \pm 1,50$} & 0,50 \\
\hline Multimistura & \multicolumn{2}{|c|}{$5,30 \pm 2,40$} & \multicolumn{2}{|c|}{$6,00 \pm 2,00$} & 0,50 \\
\hline Uma colher chá óleo no prato & \multicolumn{2}{|c|}{$3,00 \pm 0,00$} & \multicolumn{2}{|c|}{$5,50 \pm 1,80$} & 0,20 \\
\hline Papa grossa** & \multicolumn{2}{|c|}{$3,90 \pm 2,40$} & \multicolumn{2}{|c|}{$3,50 \pm 2,40$} & 0,70 \\
\hline WAZ médio & \multicolumn{2}{|c|}{$-0,31 \pm 1,21$} & $-0,7$ & $=1,29$ & $<0,001$ \\
\hline HAZ médio & $-0,0$ & 1,30 & $-0,6$ & $=1,21$ & $<0,001$ \\
\hline WHZ médio & $-0,2$ & 0,98 & $-0,4$ & $=1,17$ & 0,10 \\
\hline WAZ $<-2$ desvios padrões & 10 & 5,6 & 28 & 15,6 & 0,002 \\
\hline HAZ $<-2$ desvios padrões & 12 & 6,7 & 25 & 14,0 & 0,02 \\
\hline WHZ $<-2$ desvios padrões & 4 & 2,2 & 8 & 4,5 & 0,20 \\
\hline Hemoglobina $\mathrm{g} / \mathrm{dl}(\mathrm{n}=304)$ & 10 & 1,52 & 10, & $=1,60$ & 0,20 \\
\hline Hemoglobina $\mathrm{g} / \mathrm{dl}(\mathrm{n}=304)$ & & & & & 0,70 \\
\hline$\geq 11,0$ & 68 & 45,3 & 75 & 48,7 & \\
\hline $8-10,9$ & 72 & 48,0 & 68 & 44,2 & \\
\hline $5-7,9$ & 10 & 6,7 & 11 & 7,1 & \\
\hline
\end{tabular}

* média \pm desvio padrão; ** somente para menores de oito meses de idade $(n=26)$;

$\star \star \star$ média \pm desvio padrão entre os que consumiram pelo menos uma vez por semana.

houve diferença estatisticamente significativa quanto ao número médio de dias por semana em que cada um dos alimentos investigados era consumido. Não se observou diferença estatisticamente significativa quanto ao peso ou comprimento médio das crianças dos dois grupos ao ingressarem no estudo. O escore WAZ médio, no entanto, foi maior no grupo intervenção do que no controle $(-0,31$ versus $-0,78$; $\mathrm{p}<0,001)$. O mesmo ocorreu com relação ao es- core HAZ $(-0,03$ versus $-0,63 ; \mathrm{p}<0,001)$. A proporção de crianças do grupo intervenção com escore WAZ ou HAZ abaixo de menos dois desvios padrões para a referência foi menor do que a observada no grupo controle ( $p=0,002)$.

Foi possível coletar amostras de sangue em 304 (150 do grupo intervenção e 154 do controle) das 362 crianças. O percentual de perdas e recusas não diferiu entre os grupos $(p=0,30)$. O principal motivo das perdas foi não encon- 
trar a mãe e a criança no domicílio, após três tentativas, em dias e horários diferentes. Em ambos os grupos, a perda associou-se significativamente com o número de crianças menores de seis anos no domicílio, sendo menor nas famílias mais numerosas. Ao ingressar no estudo, $53,0 \%$ das crianças eram anêmicas $(54,7 \%$ entre as do grupo intervenção e 51,3\% entre as do grupo controles; $\mathrm{p}=0,50$ ), sendo o nível médio de hemoglobina de $10,52 \pm 1,52 \mathrm{~g} / \mathrm{dl}$ no grupo intervenção e $10,71 \pm 1,6 \mathrm{~g} / \mathrm{dl}$ no controle $(\mathrm{p}=0,20)$ (Tabela 3). A maior parte das crianças anêmicas de ambos os grupos apresentava anemia leve $(8,0-10,9 \mathrm{~g} / \mathrm{dl})$, sendo que, em toda a amostra, nenhuma apresentou anemia severa $(<5,0 \mathrm{~g} / \mathrm{dl})$.

No grupo intervenção, foi menor o número de crianças que não receberam visita da líder no mês anterior à entrevista $(27,8 \%$ versus $61,2 \%$; $<<0,001)$. Em pouco mais da metade das visitas do último mês, em ambos os grupos, a líder entrou na casa da criança para conversar com a mãe $(52,9 \%$ no grupo intervenção e $54,3 \%$ no controle; $p=0,80$ ).

Durante a visita, a criança foi vista mais freqüentemente pela líder do grupo intervenção do que pela do controle $(\mathrm{p}=0,05)$. Segundo as mães de ambos os grupos, a duração média da última visita foi de cerca de 15 minutos. Apenas 59 mães lembravam que a líder havia dado algum conselho sobre a alimentação da criança na última visita: 30 (16,9\%) do grupo intervenção e $29(16,7 \%)$ do controle $(p=1,00)$. Quando houve alguma recomendação, o uso de multimistura foi mencionado mais freqüentemente $(\mathrm{p}=0,008)$ pelas mães do grupo intervenção $(13,7 \% ; n=23)$ do que pelas do controle $(5,0 \% ; n=9)$. Acrescentar vegetais de folhas verdes à alimentação foi recomendado mais freqüentemente pelas líderes do grupo controle do que pelas do intervenção $(p=0,02)$.

$O$ percentual de perdas da primeira para a segunda visita foi de $4,1 \%$. Dentre as 347 crianças localizadas para a segunda visita (168 do grupo intervenção e 179 do controle), pôde-se coletar a amostra de sangue em 330 . As perdas associaram-se diretamente com a escolaridade do pai e com a renda familiar apenas no grupo intervenção. O nível médio de hemoglobina no grupo intervenção foi levemente superior ao observado no grupo controle, respectivamente, $10,71 \pm 1,60 \mathrm{~g} / \mathrm{dl}$ e $10,63 \pm 1,52 \mathrm{~g} / \mathrm{dl}(\mathrm{p}=0,60)$. Ao entrar no estudo, 33 crianças (21 do grupo intervenção e 12 do grupo controle) estavam recebendo sulfato ferroso. A exclusão dessas crianças da análise praticamente não mudou os resultados anteriormente obtidos, sendo a média de hemoglobina de $10,73 \pm 1,76 \mathrm{~g} / \mathrm{dl}$ no grupo intervenção e 10,61 $\pm 1,49 \mathrm{~g} / \mathrm{dl}$ no grupo controle $(\mathrm{p}=0,40)$.

Em um total de 280 crianças (135 do grupo intervenção e 145 do controle) foi possível obter as duas medidas de hemoglobina. O percentual de perdas e recusas não diferiu entre os grupos $(\mathrm{p}=0,10)$. Na Tabela 4 , a variação média do nível de hemoglobina, no período de seis meses, foi de $0,20 \pm 2,41 \mathrm{~g} / \mathrm{dl}$ no grupo intervenção e de $-0,02 \pm 1,95 \mathrm{~g} / \mathrm{dl}$ no grupo controle $(p=0,30)$. Entre as que ingressaram com hemoglobina abaixo de $11,0 \mathrm{~g} / \mathrm{dl}$, a variação foi maior entre as do grupo intervenção do que entre as do grupo controle (respectivamente, $1,28 \pm 2,22 \mathrm{~g} / \mathrm{dl}$ e $0,73 \pm 1,88 \mathrm{~g} / \mathrm{dl} ; \mathrm{p}=0,09$ ). Entre as crianças com hemoglobina $\geq 11,0 \mathrm{~g} / \mathrm{dl}$, observou-se uma diminuição média de 1,07 \pm $1,97 \mathrm{~g} / \mathrm{dl}$ no grupo intervenção, e $0,90 \pm 1,65 \mathrm{~g} / \mathrm{dl}$ no grupo controle $(\mathrm{p}=0,50)$. Excluídas as crianças em uso de sulfato ferroso ao ingressar no estudo, a variação média do nível de hemoglobina, nos seis meses de acompanhamento, foi de $0,10 \pm 2,44 \mathrm{~g} / \mathrm{dl}$ no grupo intervenção e $-0,08 \pm$ $1,92 \mathrm{~g} / \mathrm{dl}$ no grupo controle $(\mathrm{p}=0,50)$. No subgrupo de crianças com seis meses a dois anos de idade ao ingressarem no estudo, o ganho em hemoglobina foi semelhante entre os grupos $(0,94 \pm 2,43 \mathrm{~g} / \mathrm{dl}$ no grupo intervenção e $0,87 \pm$ $1,88 \mathrm{~g} / \mathrm{dl}$ no controle; $\mathrm{p}=0,80$ ).

Variação média de hemoglobina $(\mathrm{g} / \mathrm{dl})$ da primeira para a segunda visita, de acordo com os níveis no primeiro exame $(n=281)$. Pastoral da Criança, Pelotas, Rio Grande do Sul, Brasil, 2003.

\begin{tabular}{lccc}
\hline Resultado no primeiro exame & $\begin{array}{c}\text { Grupo intervenção } \\
(\mathbf{n}=136)\end{array}$ & $\begin{array}{c}\text { Grupo controle } \\
(\mathbf{n}=\mathbf{1 4 5})\end{array}$ \\
\hline Hemoglobina $<11,0 \mathrm{~g} / \mathrm{dl}$ & $1,28 \pm 2,22$ & $0,73 \pm 1,88$ & 0,09 \\
Hemoglobina $\geq 11,0 \mathrm{~g} / \mathrm{dl}$ & $-1,07 \pm 1,97$ & $-0,90 \pm 1,65$ & 0,50 \\
Todas & $0,20 \pm 2,41$ & $-0,02 \pm 1,95$ & 0,30 \\
\hline
\end{tabular}

* teste t. 
A análise por regressão linear mostrou que a intervenção promoveu uma variação bruta no nível de hemoglobina $0,23 \pm 0,26 \mathrm{~g} / \mathrm{dl}$ superior à verificada no grupo controle $(\mathrm{p}=0,30)$. Após ajuste para idade da criança, relato de sintomas respiratórios nos últimos sete dias, história de hospitalizações, escore WAZ, tipo de instalações sanitárias no domicílio e número de visitas da líder no último mês, a intervenção teve o efeito de incrementar em média 0,18 $\pm 0,27 \mathrm{~g} / \mathrm{dl}$ no nível de hemoglobina entre as crianças do grupo intervenção comparativamente às do grupo controle $(\mathrm{p}=0,50)$. De acordo com o modelo, $98,0 \%$ da variabilidade da variação média do nível de hemoglobina observada encontrava-se no nível da criança. A variabilidade no nível das líderes foi nula $(0,0 \%)$.

\section{Discussão}

Foi alta a prevalência de anemia, de 53,0\% (IC95\%: 47,2-58,7) observada entre as crianças ao ingressar no estudo, comparativamente aos achados de outros autores $12,14,15,16$, o que provavelmente reflita as más condições nutricionais das crianças das áreas periféricas da cidade de Pelotas, tipicamente o grupo alvo da ação da Pastoral da Criança.

Não houve diferença estatisticamente significativa na variação média de hemoglobina, no período de seis meses, entre as crianças dos grupos intervenção e controle. Sendo este um estudo de efetividade, onde a intervenção foi testada em condições reais de trabalho, é necessário que se considerem as razões de natureza metodológica e logística do estudo para a interpretação dos achados. Um dos aspectos diz respeito à possível contaminação do grupo controle pelos conteúdos do aconselhamento nutricional dado às líderes do grupo intervenção. As sete crianças do grupo controle, cujas mães referiram acrescentar uma colher das de chá de óleo de soja ao prato da criança, constituem uma pista da possível ocorrência dessa contaminação. Entre nove mães que referiram haver recebido da líder, na visita anterior, a recomendação de acrescentar uma colher das de chá de óleo vegetal ao prato da criança, quatro pertenciam ao grupo controle. O mesmo foi relatado durante a segunda visita do estudo: de nove mães que referiram que a líder havia recomendado acrescentar óleo vegetal ao prato da criança, três pertenciam ao grupo controle. Um segundo fato corroborando com essa hipótese decorre do treinamento inadvertido de duas líderes do grupo controle. Embora as crianças dessas líderes tenham sido excluídas do estu- do, a potencial contaminação de seus pares atuando na mesma área não pode ser descartada. Há, no entanto, a possibilidade de que essa recomendação não tenha sido dada pela líder, mas sim por médicos dos postos de saúde dessas comunidades, haja vista que esses, há cerca de cinco anos, em uma intervenção de eficácia, tinham sido treinados para o mesmo aconselhamento nutricional 7 . Tendo havido contaminação das líderes do grupo controle, o efeito esperado desse viés seria no sentido de desviar as medidas de efeito em direção à unidade, reduzindo, portanto, o real efeito da intervenção.

Além disso, em uma das comunidades do grupo intervenção, duas das líderes treinadas dividiam o acompanhamento de suas crianças com duas outras que não tinham recebido o treinamento. Como na situação anterior, tal fato pode ter resultado em enfraquecimento da intervenção, uma vez que essas foram responsáveis pelo acompanhamento de $51,4 \%$ das crianças desse grupo.

É possível ainda que a não verificação de efeito seja decorrente da falta de poder do estudo. Com o número de crianças selecionadas e os desvios padrões observados em cada grupo, o estudo tinha um poder de apenas $48,0 \%$ para detectar uma diferença $\geq 0,5 \mathrm{~g} / \mathrm{dl}$. Saliente-se, no entanto, que exceto as atendidas pelas duas líderes do grupo controle treinadas inadvertidamente, foram incluídas todas as crianças cobertas pela Pastoral da Criança em Pelotas. Se o parâmetro desvio padrão fosse conhecido de antemão, o número total de crianças elegíveis para o estudo não atenderia as necessidades de tamanho amostral. Por outro lado, a forma emparelhada como a análise foi conduzida, sendo a mesma criança medida antes e depois, exige, normalmente, um tamanho de amostra inferior ao requerido para amostras não emparelhadas 17. Além disso, a modelagem multiníveis utilizada tem, entre outras, a vantagem de otimizar o tamanho da amostra, em comparação a outros métodos 18 . No entanto, havendo a variabilidade das estimativas concentrado-se no nível das crianças $(98,0 \%)$, essa vantagem metodológica foi perdida.

Houve além disso, aparentemente, uma baixa adesão das líderes à veiculação do aconselhamento nutricional, conforme identificado pela baixa taxa de respostas positivas no recordatório materno das recomendações recebidas. A interpretação dos achados assumiu que os seguintes passos, ligando o treinamento das líderes ao desfecho, seriam necessários para que a intervenção tivesse um efeito detectável: primeiro, o material deveria ter sido adequa- 
damente adaptado à linguagem das líderes; segundo, o treinamento deveria aumentar o conhecimento das líderes sobre avaliação e aconselhamento nutricionais; terceiro, a líder deveria aderir à intervenção visitando a família e aconselhando a mãe; quarto, a mãe deveria compreender e lembrar a mensagem recebida; e quinto, a mãe deveria aderir à intervenção, modificando a alimentação da criança, o que resultaria em melhora no nível de hemoglobina no sangue capilar. Nem todos os passos dessa cadeia foram mensurados mas, pelo relato das mães, verificou-se que, embora o número de crianças que receberam visita da líder fosse maior no grupo intervenção do que no controle, as mães não lembravam ter recebido qualquer recomendação nutricional em $83,0 \%$ dos casos. Em conseqüência, as mães do grupo intervenção não modificaram a alimentação das crianças, conforme obtido pelo recordatório alimentar de 24 horas e pela freqüência semanal de consumo de alimentos, e o desfecho esperado não se verificou. Tendo sido esse um estudo sobre efetividade, nenhum procedimento adicional foi adotado para garantir que as líderes aderissem à intervenção. A possibilidade de as mães esquecerem as recomendações que tenham, de fato, recebido é improvável. O recordatório materno de recomendações nutricionais recebidas em um outro estudo conduzido em Pelotas 19 mostrou-se altamente confiável e diretamente associado ao número de conselhos recebidos.

Há um aspecto metodológico do estudo que precisa ser considerado ao analisar-se a adesão das líderes ao aconselhamento nutricional. Em ambas as visitas, a entrevistadora referia-se ao aconselhamento nutricional recebido da líder durante somente a última visita à criança, sem considerar as visitas intermediárias, ocorridas após o treinamento. O número de crianças que receberam visitas da líder no mês anterior foi significativamente maior no grupo intervenção do que no grupo controle, em ambas as entrevistas. Em função disso, a líder treinada em aconselhamento nutricional criou mais oportunidades para recomendar à mãe do que as não treinadas. É possível, portanto, que a líder tenha aconselhado a mãe em outras visitas que não apenas a imediatamente anterior à entrevista. Além disso, devido à logística do estudo, é possível que a primeira entrevista tenha ocorrido antes que a líder já treinada tenha visitado a criança, uma vez que cerca de $1 / 3$ das mães do grupo intervenção $(34,4 \%)$ foi visitada pelo estudo nos primeiros trinta dias imediatamente após o treinamento. No caso de as líderes treinadas não haverem recomendado a mãe quanto à alimentação da criança, o efeito esperado desse viés de não adesão seria igualmente no sentido de desviar o efeito da intervenção em direção à unidade, enfraquecendo seu real efeito sobre a variação do nível de hemoglobina no período.

Embora a diferença na variação média da hemoglobina entre os dois grupos não tenha sido estatisticamente significativa, essa foi positiva no grupo intervenção e negativa no controle. De fato, o consumo de ferro na dieta das 24 horas anteriores a segunda entrevista, foi significativamente maior entre as crianças do grupo intervenção do que entre as do grupo controle (respectivamente, 9,35 $\pm 4,45 \mathrm{mg}$ versus $8,37 \pm 4,64 \mathrm{mg} ; \mathrm{p}=0,04)$. Embora o consumo seja apenas o do dia anterior à entrevista, tal achado, juntamente com a variação positiva da hemoglobina nesse grupo, podem estar indicando um consumo de ferro realmente maior no grupo cujas líderes foram treinadas em aconselhamento nutricional. O fenômeno de regressão à média, possível de ser verificado em função dos níveis iniciais de hemoglobina observados, não justifica a diferença detectada, uma vez que as médias de hemoglobina e a proporção de crianças abaixo de $11,0 \mathrm{~g} / \mathrm{dl}$ na primeira medida, bem como a proporção de crianças recebendo sulfato de ferro ao ingressar no estudo foram estatisticamente semelhantes nos dois grupos. Nenhuma das 36 crianças que recebiam sulfato ferroso ao entrar no estudo continuava em uso por ocasião da segunda visita. Além disso, somente duas crianças, ambas do grupo intervenção, passaram a receber sulfato ferroso entre a primeira e a segunda visitas.

Idealmente, o aconselhamento nutricional deveria prevenir a deficiência de ferro. A proporção de crianças que se tornaram anêmicas no período de acompanhamento, no entanto, foi semelhante entre os grupos intervenção e controle. Sendo a anemia a manifestação mais tardia e grave da deficiência de ferro, é provável que o aconselhamento nutricional tenha ocorrido em uma fase da carência do mineral que a alimentação, isoladamente, seria incapaz de reverter. Assim, seria recomendável que as líderes direcionassem a intervenção nutricional prioritariamente às mães de crianças no primeiro ano de vida, de forma a prevenir o esgotamento das reservas de ferro até seu estágio mais grave, a anemia carencial.

Seria interessante que este estudo fosse replicado em outros locais, particularmente onde a cobertura da Pastoral da Criança pudesse garantir um tamanho de amostra que limitasse a dispersão do nível de hemoglobina. Seria im- 
portante também iniciar a intervenção recrutando crianças nos primeiros seis meses de vida, uma vez que, no atual estudo, somente $23,7 \%$ eram amamentadas à época do arrolamento.

\section{Resumo}

Para avaliar a efetividade do treinamento das líderes da Pastoral da Criança em aconselhamento nutricional sobre a variação da hemoglobina entre menores de seis anos, foi realizada uma intervenção comunitária. Por sorteio, uma das duas áreas de ação da Pastoral da Criança em Pelotas, Rio Grande do Sul, Brasil, foi selecionada e as líderes treinadas (Grupo Intervenção). A outra área constituiu o Grupo Controle. Após consentimento, a mãe foi entrevistada sobre a família, saúde e alimentação da criança. A hemoglobina foi medida com fotômetro portátil ao ingressar no estudo e após seis meses. O desfecho foi a variação da hemoglobina, da primeira para a segunda medida. Ingressaram 183 crianças intervenção e 179 crianças controle, comparáveis quanto ao sexo, idade, características ao nascer, aporte de ferro e nível médio de hemoglobina. Na análise ajustada, a variação no grupo intervenção foi 0,18 $\pm 0,27 \mathrm{~g} / \mathrm{dl}$ maior do que no controle. Embora a diferença entre os grupos não fosse estatisticamente significativa, a variação foi positiva no grupo intervenção e negativa no grupo controle, sugerindo benefício do treinamento.

Deficiência de Ferro; Anemia Ferropriva; Nutrição da Criança

\section{Colaboradores}

I. Santos coordenou a equipe de investigadores, tendo participado de todas as fases do estudo, desde o planejamento, elaboração de instrumentos, treinamento das líderes da Pastoral da Criança, análise dos dados, interpretação dos achados e redação final do manuscrito. J. A. César participou do planejamento do estudo e contribuiu na interpretação dos resultados. G. Minten auxiliou na elaboração dos instrumentos, no treinamento das líderes da Pastoral da Criança e das entrevistadoras, coordenou o trabalho de campo, a entrada e edição dos dados, realizou as análises preliminares e contribuiu na interpretação dos achados. P. L. Marco revisou os questionários antes da digitação, auxiliou na checagem da amplitude e consistência do banco, na edição dos dados e nas análises brutas. N. Valle colaborou na fase de análise estatística dos dados.

\section{Agradecimentos}

Os autores agradecem o apoio financeiro do Banco Itaú Social, através da Pastoral da Criança do Brasil, aos coordenadores e às líderes da Pastoral da Criança em Pelotas e a todas as mães e crianças, que tornaram possível a realização deste estudo. 


\section{Referências}

1. Stoltzfus RJ. Defining iron-deficiency anemia in public health terms: a time for reflection. J Nutr 2001; 131 (2 Suppl 2):565S-7S.

2. Beard JL. Iron biology in immune function, muscle metabolism and neuronal functioning. J Nutr 2001; 131 (2 Suppl 2):568S-80S.

3. Grantham-McGregor S, Ani C. A review of studies on the effect of iron deficiency on cognitive development in children. J Nutr 2001; 131 (2 Suppl 2):649S-68S

4. Lozoff B, Jimenez E, Hagen J, Mollen E, Wolf AW. Poorer behavioral and developmental outcome more than 10 years after treatment for iron deficiency in infancy. Pediatrics 2000; 105:E51.

5. Gillespie S, Johnston JL. Expert consultation on anemia determinants and interventions. Ottawa: Micronutrient Initiative Association; 1998.

6. Victora CG, Habicht JP, Bryce J. Evidence-based public health: moving beyond randomized trials. Am J Public Health 2004; 94:400-5.

7. Santos I, Victora CG, Martines J, Gonçalves H, Gigante DP, Valle NJ, et al. Nutrition counseling increases weight gain among Brazilian children. J Nutr 2001; 131:2866-73.

8. Valle NJ, Santos I, Gigante DP, Gonçalves H, Victora CG, Martines J, et al. Household trials with very small samples predict responses to nutrition counseling intervention. Food Nutr Bull 2003; 24: 343-9.

9. World Health Organization. Management of childhood illness. Counsel the mother. Geneva: Division for Control of Diarrhoeal and Respiratory, World Health Organization; 1995.

10. World Health Organization/United Nations Children's Fund. Management of childhood illness. Management of the sick young infant age 1 week up to 2 months. Geneva: Division for Control of Diarrhoeal and Respiratory, World Health Organization; 1995.
11. Santos I, Victora CG, Martines J, Gonçalves H, Gigante DP, Valle NJ, et al. Avaliação da eficácia do aconselhamento nutricional dentro da estratégia do AIDPI (OMS/UNICEF). Rev Bras Epidemiol 2002; 5:15-29.

12. Osório MM, Lira PIC, Batista-Filho M, Ashworth A. Prevalence of anemia in children 6-59 months old in the state of Pernambuco, Brazil. Rev Panam Salud Pública 2001; 10:101-7.

13. Baker SJ. Nutritional anemia: a major controlable public health problem. Bull World Health Organ 1978; 56:659-75.

14. Monteiro CA, Sczarfarc SC. Estudo das condições de saúde das crianças no Município de São Paulo, SP (Brasil), 1984-1985. V - Anemia. Rev Saúde Pública 1987; 21:255-60.

15. Oliveira RS, Diniz AS, Benigna MJC, Miranda-Silva SM, Lola MM, Gonçalves MC, et al. Magnitude distribuição espacial e tendência da anemia em pré-escolares da Paraíba. Rev Saúde Pública 2002; 36:26-32.

16. Silva LSM, Giugliani ERJ, Aerts DRGC. Prevalência e determinantes de anemia em crianças de Porto Alegre, RS, Brasil. Rev Saúde Pública 2001; 35:66-73.

17. Hulley SB, Cummings SR. Designing clinical research: an epidemiological approach. Baltimore: Williams \& Wilkins; 1988

18. Goldstein H. Multilevel statistical models. London: Edward Arnorld; 1995.

19. Pelto GH, Santos I, Gonçalves H, Victora C, Martines J, Habicht JP. Nutrition counseling training changes physician behavior and improves caregiver knowledge acquisition. J Nutr 2004; 134: 357-62.

Recebido em 26/Fev/2004

Versão final reapresentada em 19/Mai/2004 Aprovado em 30/Jul/2004 